\title{
Transient MHD Free Convective Flow of an Optically Thick Gray Gas Past a Moving Vertical Plate in the Presence of Thermal Radiation and Mass Diffusion
}

\author{
S. K. Ghosh ${ }^{1 \dagger}$, S. Das ${ }^{2}$ and R. N. Jana ${ }^{3}$ \\ ${ }^{1}$ Department of Mathematics, Narajole Raj Collge, Narajole 721211, West Bengal, India \\ ${ }^{2}$ Department of Mathematics, University of Gour Bnaga, Malda 732 103, West Bengal, India \\ ${ }^{3}$ Department of Applied Mathematics, Vidyasagar University, Midnapore 721 102, West Bengal, India \\ $\dagger$ Corresponding Author Email: g_swapan2002@yahoo.com
}

(Received December 7, 2013; accepted February 5, 2014)

\begin{abstract}
The purpose of present investigation is to analyse bouyancy-driven radiation-convection flow past a moving vertical plate with reference to an optically dense medium in the presence of mass concentration, using Rosseland approximation permeated by a magnetic field. The flow is considered to be gray in the presence of free convection, mass transfer and radiation. An exact solution of the governing equations is obtained by applying the Laplace transform method. Numerical results of velocity distributions, shear stress, temperature distribution and mass concentration are presented graphically to give physical insight into the flow pattern.
\end{abstract}

Keywords: MHD flow, Free convection. Mass transfer, Radiation, Gray gas and Mass diffusion.

\section{NOMENCLATURE}

$\begin{array}{ll}\vec{B} & \text { magnetic field vector } \\ B_{0} & \text { magnetic flux intensity } \\ c_{p} & \text { specific heat at constant pressure } \\ \vec{E} & \text { electric field vector } \\ F & \text { chemical molecular diffusivity } \\ \hat{g} & \text { acceleration due to gravity } \\ G r & \text { Grashof number } \\ G m & \text { modified Grashof number } \\ \vec{J} & \text { current density vector } \\ \text { Le } & \text { Lewis-Semenov number } \\ k^{*} & \text { spectral mean absorption coefficient } \\ k & \text { thermal conductivity } \\ k_{1} & \text { radiation parameter } \\ \beta & \text { coefficient of thermal expansion } \\ \beta^{*} & \text { coefficient of thermal expansion with concentration } \\ v & \text { kinematic viscosity } \\ \phi & \text { non-dimensional Species concentration } \\ \phi^{\prime} & \text { species concentration } \\ \phi_{w}^{\prime} & \text { species concentration at the plate } \\ \phi_{\infty}^{\prime} & \text { concentration far away from the plate }\end{array}$

$\begin{array}{cl}M^{2} & \text { Hartmann number } \\ \operatorname{Pr} & \text { Prandtl number } \\ q_{r} & \text { radiative heat flux } \\ \vec{q} & \text { velocity field vector } \\ S c & \text { Schimdt number } \\ T & \text { dimensionless fluid temperature } \\ T^{\prime} & \text { fluid temperature } \\ T_{w}^{\prime} & \text { temperature at the plate } \\ T_{\infty}^{\prime} & \text { temperature of the fluid far away from the plate } \\ U & \text { uniform plate velocity } \\ u^{\prime} & \text { fluid velocity along the plate } \\ u & \text { dimensionless fluid velocity } \\ (x, y) & \text { Cartesian co-ordinates } \\ \mu_{e} & \text { magnetic permeability } \\ \rho & \text { fluid density } \\ \sigma & \text { electrical conductivity of fluid } \\ \sigma^{*} & \text { Stefan-Boltzman constant } \\ \tau & \text { non-dimensional time } \\ \tau_{0} & \text { shear stress at the plate }\end{array}$




\section{INTRODUCTION}

High temperature thermal radiation of an optically thick gray gas becomes significant to the relevance of space technology in such way that the foundation of space laboratory in the zero gravity is established by the concept of gray body radiation. If the temperature of surrounding fluid is rather high, radiation effects play an important role and this situation does not exist in space technology. In such cases one has to take into account the effect of thermal radiation and mass diffusion. It takes place in numerous industrial applications, e.g. polymer production, manufacturing of ceramics or glassware, and food processing Cussler (1998). For some industrial applications such as glass production, furnace design, propulsion systems, plasma physics and spacecraft re-entry aerothermodynamics which operate at higher temperatures and radiation effect can also be significant. A clear understanding of the nature of interaction between thermal and concentration buoyancies is necessary. Consolidated effects of heat and mass transfer problems are of importance in many chemical formulations and reactive chemicals. Therefore, considerable attention had been paid in recent years to study the influence of the participating parameters on the velocity field. More such engineering application can be seeing in electrical power generation systems when the electrical energy is extracted directly from a moving conducting fluid.

The study of magnetohydrodynamic flow for electrically conducting fluid past heated surface has attracted the interest of many researches in view of its important applications in many engineering problems such as plasma studies, petroleum industries MHD power generations, cooling of nuclear reactors, the boundary layer control in aerodynamics and crystal growth. Until recently this study has been largely concerned with flow and heat transfer characteristics in various physical situations. Radiative magnetohydrodynamic flows arise in many areas of technology and applied physics including oxide melt materials processing (Shu et al. (2004)), astrophysical fluid dynamics (Stone and Norman (1992); Vishwakarma et al. (1987)), plasma flow switch performance (Bowers et al. (1990)), MHD energy pumps operating at very high temperatures (Biberman et al.(1979)) and hypersonic aerodynamics (Ram and Pandey (1980)). Takhar et al. (1996) investigated the effects of radiation on the MHD free convection flow of radiating gas past a semi-infinite vertical plate. Raptis and Masslas (1998) studied unsteady magnetohydrodynamics convection in a gray, absorbingemitting but non-scattering fluid regime using the Rosseland radiation model. A similar study was communicated by Raptis and Perdikis(2000). Azzam (2002) considered thermal radiation flux influence on hydromagnetic mixed convective steady optically-thick laminar boundary layer flow also using Rosseland approximation. Helliwell and Mosa (1979) reported on thermal radiation effects in buoyancy-driven hydromagnetic flow in a horizontal channel flow with an axial temperature gradient in the presence of Joule and Viscous heating. Yasar and Moses (1992) developed a one dimensional adaptive-grid finite-differencing computer code for thermal radiation magnetohydrodynamic simulation of fusion plasmas. Excellent studies of thermal radiation-convection magnetohydrodynamics include Duwairi and Damseh (2004), Raptis et al. (2004) who considered axisymmetric flow and Duwairi and Duwairi (2005) who studied thermal radiation heat transfer effects on the hydrodynamic Rayleigh flow of a gray viscous fluid. Aboeldahab and Azzam(2005) have described the effects of magnetic field on hydromagnetic mixed free forced heat and mass convection of a gray, optically-thick, electrically conducting viscous fluid along a semi-infinite inclined plane for high temperature and concentration using the Rosseland approximation. Ghosh and Pop (2007) and Jana and Ghosh (2011) have studied thermal radiation of an optically-thick gray gas in the presence of indirect natural convection showing that the pressure rise region leads to slightly increase in the velocity with an increase of radiation parameter. Patra et.al (2012) considered transient approach to radiation heat transfer free convection flow with ramped wall temperature. Rajput and Kumar (2012) examined the radiation effects on MHD flow past an impulsively started vertical plate with variable heat and mass transfer. Ahmed and Kalita (2013) presented an analytical and numerical study for MHD radiating flow over an infinite vertical surface bounded by a porous medium in presence of chemical reaction. Al-Odat and Al-Azab (2007) have examined the influence of chemical reaction on transient MHD free convection over a moving vertical plate. Mbeledogu and Ogulu (2007) have presented the heat and mass transfer of an unsteady MHD natural convection flow of a rotating fluid past a vertical porous plate in the presence of radiative heat transfer. The MHD transient free convection and chemically reactive flow pasta porous vertical plate with radiation and temperature gradient dependent heat source in slip flow regime have been studied by Rao et al. (2013). Reddy et al. (2012) have investigated the heat and mass transfer effects on unsteady MHD free convection flow past a vertical permeable moving plate with radiation.

The aim of present investigation is to analyze the influence of a magnetic field on buoyancy-driven radiation-convection flow of an optically dense medium with mass concentration, using the Rosseland approximation. The orientation of the present problem leads to a gray body radiation resulting from space application to generate X-ray photon in a vacuum. Since the Sun is exposed to a vacuum, X-ray photon is liberated from the Sun with reference to gray body radiation. Gray body radiation in the presence of a magnetic field is subjected to a non-catalytic system in such a way that the homogeneous reaction is confined with equal concentration of ions and electrons. The homogeneous reaction takes place in an equilibrium condition with regard to the rate of heat and mass transfer. A strong magnetic field provides for effective thermal insulation of hot plasma. In this situation, the rate of temperature distribution and mass concentration at wall become important to a study of gray body radiation in the presence of a thin radiation layer at the wall. The mode of present investigation is to study the 
unsteady magnetohydrodynamic flow of viscous incompressible electrically conducting fluid occupying a semi-infinite region of space bounded by an infinitevertical moving plate. The flow is considered a gray gas, optically thick, absorbing-emitting but nonscattering in the presence of free convection, mass transfer and radiation. Such a study has to the authors' knowledge not appeared in the scientific literature. An exact solution of the governing equations is obtained in a closed form by employing Laplace transform technique. The effects of the magnetic parameter (Hartmann number, $M^{2}$ ), free convection parameter (Grashof number i.e. $G r$ ), modified Grashof number with concentration $(G m)$, mass concentration $(\phi)$, time $(T)$ and the radiation parameter $\left(k_{1}\right)$ on velocity distribution and shear stresses at the plate are presented graphically. An excellent agreement with the relation of heat and mass transfer is established in such a way that the ratio of concentration and temperature field is dependent on the radiation parameter if their rates are equal. On the other hand, the concentration and temperature field will be similar to the absence of radiation parameter $k_{1}$ and its ratio is known as the Lewis-Semenov number. The novelty of this study is to determine the Lewis-Semenov number in the absence of radiation parameter.

\section{Formulation of The Problem and its SOLUTION}

Consider an unsteady magnetohydrodynamic flow of a viscous incompressible electrically conducting fluid occupying a semi-infinite region of space bounded by an infinite vertical moving plate. It is considered that the velocity of the fluid far away from the plate is zero. The flow is considered in an opticlly thick gray gas with the decisive importance to free convection, mass transfer and radiation. We choose the co-ordinate system in such a way that the $x^{\prime}$ - axis is taken along the plate and the $y^{\prime}$-axis is normal to it. A uniform magnetic field is applied parallel to $y^{\prime}$-axis (see Fig.1). It is considered that all the fluid properties are constant except the influence of the density variation on the body force term. The radiative heat flux in the $x^{\prime}$-direction is considered negligible in comparison to the $y^{\prime}$ direction.

Under Boussinesq approximation, the MHD equation of motion becomes

$$
\begin{aligned}
& \frac{\partial \vec{q}}{\partial t}+(\vec{q} \cdot \nabla) \vec{q}=-\frac{1}{\rho} \nabla p+v \nabla^{2} \vec{q}+\frac{1}{\rho} \vec{J} \times \vec{B} \\
& +\hat{g} \beta\left(T^{\prime}-T_{\infty}^{\prime}\right)+\hat{g} \beta^{*}\left(\phi^{\prime}-\phi_{\infty}^{\prime}\right) .
\end{aligned}
$$

The equation of continuity becomes

$$
\nabla \cdot \vec{q}=0 .
$$

The Ohm's law for a moving conductor

$$
\vec{J}=\sigma(\vec{E}+\vec{q} \times \vec{B}) .
$$

The Maxwell's equations are

$$
\nabla \times \vec{B}=\mu_{e} \vec{J},
$$

$\nabla \times \vec{E}=-\frac{\partial \vec{B}}{\partial t}$,

$\nabla \cdot \vec{B}=0$ (Solenoidal relation)

$\nabla \cdot \vec{J}=0($ Conservation of electric charge $)$

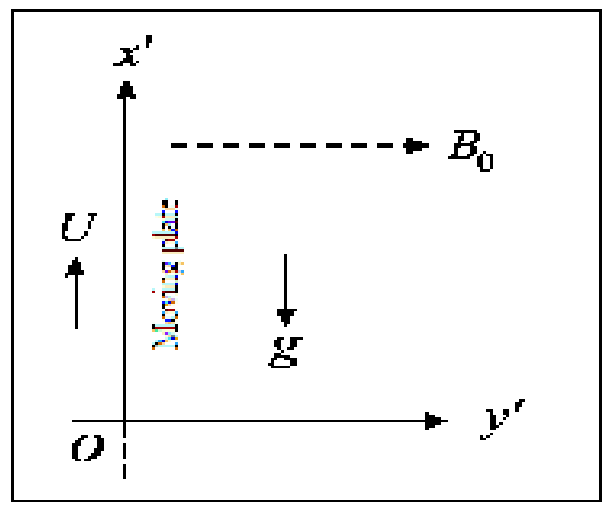

Fig. 1. Geometry of the problem

The following equation is in agreement with the fundamental eqautions of magnetohydrodynamics (1)(4)

$$
\begin{aligned}
\frac{\partial u^{\prime}}{\partial t} & =v \frac{\partial^{2} u^{\prime}}{\partial y^{\prime 2}}+g \beta\left(T^{\prime}-T_{\infty}^{\prime}\right) \\
& +g \beta^{*}\left(\phi^{\prime}-\phi_{\infty}^{\prime}\right),-\frac{\sigma B_{0}^{2}}{\rho} u^{\prime}
\end{aligned}
$$

The equation of energy transfer is

$$
\frac{\partial T^{\prime}}{\partial t}=\frac{k}{\rho c_{p}} \frac{\partial^{2} T^{\prime}}{\partial y^{\prime 2}}-\frac{1}{\rho c_{p}} \frac{\partial q_{r}}{\partial y^{\prime}}
$$

The equation of mass transfer is

$$
\frac{\partial \phi^{\prime}}{\partial t}=F \frac{\partial^{2} \phi^{\prime}}{\partial y^{\prime 2}}
$$

where $\vec{B}, \vec{E}, \vec{q}, \vec{J}, \mu_{e}$ are respectively, the magnetic field vector, the electric field vector, the fluid velocity vector, the current density vector and the magnetic permeability. $u^{\prime}, t, v, \hat{g}, \beta, \beta^{*}, \phi^{\prime}, \phi_{\infty}^{\prime}$ , $\quad T^{\prime}, \quad T_{\infty}^{\prime}, k, \quad c_{p}, \rho, \sigma, \quad B_{0}, \quad F$ and $q_{r}$ are respectively, the velocity component along the plate, the time, the kinematic viscosity, the gravitational acceleration, the coefficient of thermal expansion, the coefficient of thermal expansion with concentration, the species contration, the concentration far away from the plate, the temperature of the fluid, the temperature of the fluid far away from the plate, the thermal conductivity, the specific heat at constant pressure, the fluid density, the electrical conductivity, the magnetic flux intensity, the chemical molecular diffusivity and radiative heat flux.

In general, the electri current flowing in the fluid gives rise to an induced magnetic field, which will distort the applied magnetic field. Since the magnetic Reynolds number is very small for partially ionized fluid, the induced magnetic field produced by the motion of an electric field is applied, the polarization voltage is negligible i.e. the electric field vector $\vec{E}$ is absent (see 
Maryer, (1958)). This corresponds to the case where no energy is being added or extracted from the fluid by electrical means. The magnetic effects are confined to retarding the flow and dissipating energy of motion into heat. Since radiation layer is thin, the concentration and temperature flied depend on the radiation parameter.

The initial and the boundary conditions for velocity and temperature distribution are

$u^{\prime}=0, T^{\prime}=T_{\infty}^{\prime}, \phi^{\prime}=\phi_{\infty}^{\prime}$ for all $y^{\prime} \geq 0$ and $t \leq 0$,

$u^{\prime}=U, T^{\prime}=T_{w}^{\prime}, \phi^{\prime}=\phi_{w}^{\prime}$ at $y^{\prime}=0$ for $t>0$,

$u^{\prime} \rightarrow 0, T^{\prime} \rightarrow T_{\infty}^{\prime}, \phi^{\prime} \rightarrow \phi_{\infty}^{\prime} \quad$ as $y^{\prime} \rightarrow \infty$ for $t>0$,

where $T_{w}^{\prime}$ is the temperature at the plate, $\phi_{w}^{\prime}$ the species concentration at the plate and $U$ the velocity of the plate.

The radiative heat flux is addressed using the approach outlined by Isachenko et al. (1969). The Rosseland diffusion flux approximation is therefore used leading to a Fourier-type gradient function, viz:

$q_{r}=-\frac{4 \sigma^{*}}{3 k^{*}} \frac{\partial T^{\prime 4}}{\partial y^{\prime}}$,

where $k^{*}$ is the spectral mean absorption coefficient of the medium and $\sigma^{*}$ the Stefan-Boltzman constant.

It is assumed that the temperature differences within the flow are sufficiently small such that $T^{\prime 4}$ may be regarded as a linear function of the temperature. It can be established by expanding $T^{\prime 4}$ in a Taylor series about $T_{\infty}^{\prime}$ and neglecting higher order terms. Therefore, $T^{\prime 4}$ can be expressed in the following form

$T^{\prime 4}=4 T_{\infty}^{\prime 3} T^{\prime}-3 T_{\infty}^{\prime 4}$.

Introducing dimensionless quantities

$$
\begin{aligned}
& y=\frac{y^{\prime} U}{v}, \tau=\frac{t U^{2}}{v}, u_{1}=\frac{u}{U_{0}}, \\
& T=\frac{T^{\prime}-T_{\infty}^{\prime}}{T_{w}^{\prime}-T_{\infty}^{\prime}}, \phi=\frac{\phi^{\prime}-\phi_{\infty}^{\prime}}{\phi_{w}^{\prime}-\phi_{\infty}^{\prime}}, \\
& G r=\frac{g \beta v\left(T_{w}^{\prime}-T_{\infty}^{\prime}\right)}{U^{3}}, G m=\frac{g \beta^{*} v\left(\phi_{w}^{\prime}-\phi_{\infty}^{\prime}\right)}{U^{3}} \\
& M^{2}=\frac{\sigma v B_{0}^{2}}{\rho U^{2}} \quad \operatorname{Pr}=\frac{\rho v c_{p}}{k}, S c=\frac{v}{F},
\end{aligned}
$$

where $\phi, G r, G m, M^{2}, \operatorname{Pr}, S c \quad$ are respectively concentration, Grashof number, modified Grashof number, Hartmann number, the Prandtl number and Schimdt number.

Using Eqs.(9) and (10), the energy Eq.(6) can be written in a dimensionless form subject to Eq.(11) such as

$$
\left(1+k_{1}\right) \frac{\partial^{2} T}{\partial y^{2}}-\operatorname{Pr} \frac{\partial T}{\partial \tau}=0
$$

where $k_{1}=\frac{16 \sigma^{*} T_{\infty}^{\prime 3}}{3 k^{*} k}$ is the radiation parameter.

Using Eq.(11), Eqs.(5) and (7) transform into a dimensionless form as

$$
\begin{aligned}
& \frac{\partial u}{\partial \tau}+M^{2} u=\frac{\partial^{2} u}{\partial y^{2}}+T G r+\phi G m, \\
& \frac{\partial^{2} \phi}{\partial y^{2}}-S c \frac{\partial \phi}{\partial \tau}=0,
\end{aligned}
$$

The corresponding initial and boundary conditions for $u$ and $\theta$ are

$$
\begin{aligned}
& u=0, T=0, \phi=0 \text { for all } y \geq 0 \text { and } \tau \leq 0, \\
& u=1, T=1, \phi=1 \text { at } y=0 \text { for } \tau>0, \\
& u \rightarrow 0, T \rightarrow 0, \phi \rightarrow 0 \text { as } y \rightarrow \infty \text { for } \tau>0 .
\end{aligned}
$$

Solutions for the velocity distribution, temperature distribution, mass concentration can be obtained by employing he Laplace transform technique subject to the Eqs.(12), (13) and (14) become

$$
\begin{aligned}
& u(y, \tau)=\frac{1}{2}\left(1-\frac{G r}{M^{2}}-\frac{G m}{M^{2}}\right) \\
& \times\left[e^{M y} \operatorname{erfc}\left(\frac{y}{2 \sqrt{\tau}}+M \sqrt{\tau}\right)\right. \\
& \left.+e^{-M y} \operatorname{erfc}\left(\frac{y}{2 \sqrt{\tau}}-M \sqrt{\tau}\right)\right] \\
& +\frac{G r}{2 M^{2}}\left[e^{C \tau}\left\{F_{1}\left(y, C, M^{2}, \tau\right)-F_{2}(y, C, \alpha, \tau)\right\}\right. \\
& \left.+2 F_{3}(y, \alpha, \tau)\right] \\
& +\frac{G m}{2 M^{2}}\left[e^{D \tau}\left\{F_{1}\left(y, D, M^{2}, \tau\right)-F_{2}(y, D, S c, \tau)\right\}\right. \\
& \left.+2 F_{3}(y, S c, \tau)\right] \\
& T(y, \tau)=\operatorname{erfc}\left(\frac{y}{2} \sqrt{\frac{\alpha}{\tau}}\right) \\
& \phi(y, \tau)=\operatorname{erfc}\left(\frac{y}{2} \sqrt{\frac{S c}{\tau}}\right),
\end{aligned}
$$

where

$$
\begin{aligned}
& \begin{array}{l}
F_{1}\left(x, z, M^{2}, \tau\right)=e^{x \sqrt{M^{2}+z}} \operatorname{erfc}\left(\frac{x}{2 \sqrt{\tau}}+\sqrt{\left(M^{2}+z\right) \tau}\right) \\
+e^{-x \sqrt{M^{2}+z}} \operatorname{erfc}\left(\frac{x}{2 \sqrt{\tau}}-\sqrt{\left(M^{2}+z\right) \tau}\right)
\end{array} \\
& \begin{array}{l}
F_{2}(x, z, \xi, \tau) \\
=e^{x \sqrt{z \xi}} \operatorname{erfc}\left(\frac{x}{2} \sqrt{\frac{\xi}{\tau}}+z \sqrt{\tau}\right) \\
+e^{-x \sqrt{z \xi}} \operatorname{erfc}\left(\frac{x}{2} \sqrt{\frac{\xi}{\tau}}-z \sqrt{\tau}\right)
\end{array} \\
& F_{3}(x, \xi, \tau)=\operatorname{erfc}\left(\frac{x}{2} \sqrt{\frac{\xi}{\tau}}\right) \\
& C=\frac{M^{2}}{\alpha-1}, \quad D=\frac{M^{2}}{S c-1}, \alpha=\frac{\operatorname{Pr}}{1+k_{1}} \\
& \text { and erfc (.) is the complementary error function. } \\
& \text { Solution (16) exists for Pr }>1+k_{1} .
\end{aligned}
$$


From the physical point of view, it is necessary to know the shear stress at the moving plate. The nondimensional shear stress at the plate $y=0$ is

$$
\begin{aligned}
& \tau_{0}=\left(\frac{\partial u}{\partial y}\right)_{y=0}=\left(\frac{G m}{M^{2}}-\frac{G r}{M^{2}}-1\right) \\
& \times\left[M \operatorname{erf}(M \sqrt{\tau})+\frac{1}{\sqrt{\pi \tau}} e^{-M^{2} \tau}\right] \\
& -\frac{G r}{M^{2}}\left[e^{C \tau}\left\{G_{1}\left(C, M^{2}, \tau\right)-G_{2}(C, \alpha, \tau)\right\}\right. \\
& \left.+G_{3}(\alpha, \tau)\right] \\
& -\frac{G m}{M^{2}}\left[e^{D \tau}\left\{G_{1}\left(D, M^{2}, \tau\right)-G_{2}(D, S c, \tau)\right\}\right. \\
& \left.+G_{3}(S c, \tau)\right]
\end{aligned}
$$

where

$$
\begin{aligned}
& G_{1}\left(z, M^{2}, \tau\right)=\sqrt{M^{2}+z} \operatorname{erf}\left(\sqrt{\left(M^{2}+z\right) \tau}\right) \\
& +\frac{1}{\sqrt{\pi \tau}} e^{-\left(M^{2}+z\right) \tau} \\
& G_{2}(z, \xi, \tau)=\sqrt{\xi z} \operatorname{erf}(z \sqrt{\tau})+\sqrt{\frac{\xi}{\pi \tau}} e^{-z^{2} \tau} \\
& G_{3}(\xi, \tau)=\sqrt{\frac{\xi}{\pi \tau}}
\end{aligned}
$$

Differentiating Eqs.(17) and (18) with respect to $y$, the following condition is satified

$$
\frac{\operatorname{Pr}}{1+k_{1}}=S c \text {. }
$$

It is evident from the condition (22) that the ratio of the concentration and temperature field is dependent on the radiation parameter $k_{1}$. This situation reveals that the enthalpy and molecular diffusion are closely connected and are interdependent of a chemical reaction is involved in this process. In a non-catalytic system the enthalpy can not be taken into account so that the chemical reaction in not involved in this precess of heat and mass tranfers. It is important to note that in the absence of the radiation parameter $k_{1}$, the concentartion and temperature fileld are similar if $\operatorname{Pr}=S c$ or $\frac{\operatorname{Pr}}{S c}=1$. The ratio is known as the LewisSemenov number and is denoted by $L e$.

\section{RESUlts and DISCUSSION}

To determine the physical insight into the MHD flow pattern the velocity distributions, shear stress, temperature distribution and concentration are depicted graphically in Figs.2-15 for several values of $M^{2}, G r$, $G_{m}, k_{1}, S_{c}$ and $\tau$. In Fig.2 we have presented the influence of Hartmann number $\left(M^{2}\right)$ on the velocity distribution. With increasing magnetic field strength $B_{0}$,

$M^{2}$ is increased and this serves to decelerate the flow along the plate. In accordance with this we observe in Fig.2 that $u$ profile values are strongly reduced with increasing $M^{2}$.We also note that as $M^{2}$ rises, the profile decay to zero progressively for shorter distance from the plate surface. The strong inhibiting effect of magnetic field is therefore evident. Fig.3 reveals that with an increase of radiation parameter $k_{1}$ the fluid velocity $(u)$ slightly increases in accordance with Stefan-Boltzman constant. Larger $\left(k_{1}\right)$ values correspond to an increased dominance of thermal radiation over conduction. As such thermal radiation supplements the thermal diffusion and increases the overall thermal diffusivity of the regime since the local radiant diffusion flux model adds radiation conductivity to the conventional thermal conductivity. As a result the fluid temperature and velocity in the fluid regime flow are increased. It is evident from Fig.4 that an increase in free convection parameter i.e. Grashof number $(G r)$ is found to increase velocity values. Free convection currents as stimulated with the buoyancy term to accelerate the flow along the plate. Fig.5 shows that an increase in modified Grashof number with concentration $(\mathrm{Gm})$ the flow velocity ( $u$ ) leads to increase with reference to buoyancy force in concentration field. It seems to understand that the concentration plays an important role to stabilize the flow situation. It is noticed from Fig.6 that the flow velocity $(u)$ leads to decrease with an increase in Schmidt number $(S c)$. In a non-catalytic system, the effects of concentration on velocity field give rise to fall the flow situation for effective molecular diffusion. Fig.7 demonstrates the flow velocity $(u)$ increases with the increase in time $(\tau)$ and the profiles decay to zero for shorter distance from the plate surface.

Fig.8 demonstrates that the frictional shearing stress ( $\left.\tau_{0}\right)$ at the plate decreases with increase in radiation parameter $k_{1}$. Since viscosity initiates thermal radiation there arise a destabilizing influence on shear stress at the plate. It is seen from Fig.9 that the shear stress increases with increase in Grashof number $(G r)$. This situation reveals that the buoyancy force accelerates the flow particle with stronger magnetic field leads to increase the shear stress. Fig.10 shows that the frictional shear stress $\left(\tau_{0}\right)$ decreases with increase in modified Grashof number with concentration ( $G m$ ). In the presence of a strong magnetic field, molecular diffusion retarded the frictional shearing stress with a decisive importance to a buoyancy effect with concentration. It is noticed from Fig.11 that the influence of Schmidt number ( $S c$ ) leads to rise the frictional shear stress. Since concentration plays a significant role on Schmidt number if reveals that the reduction of viscosity communicates the effect of Schmidt number under the influence of a strong magnetic field to increase the frictional shear stress.

Fig.12 reveals that the temperature field ( $T$ ) increases with increase in radiation parameter $\left(k_{1}\right)$. In an optically dense medium using Rosseland approximation, the effect of magnetic field lies in its behavior of a thin radiation layer near the plate in such a way that a magnetic field generates thermal radiation due to the presence of viscosity to boost up temperature field on increasing radiation parameter. Fig. 13 shows that with the increase in Prandtl number $(P r)$ the temperature field $(T)$ decreases near the plate. Fig.14 shows that the temperature field $(T)$ and mass concentration $(\phi)$ increase with increase in time $(\tau)$. Since the temperature 
field and the molecular diffusion depend on radiation parameter, they are gradually increase with increase in time and the effect of magnetic field become predominant. Fig.15 demonstrates that the mass concentration $(\phi)$ decreases with increase in Schmidt number. In this context, the effect of magnetic field determines the behavior of mass concentration on velocity field, shear stress and temperature field.

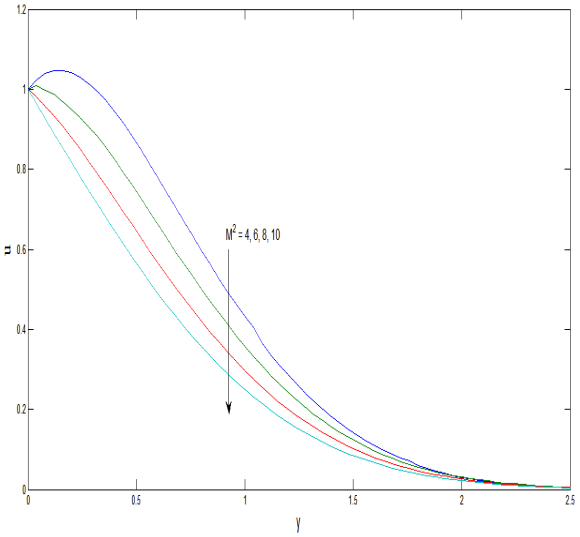

Fig. 2. Velocity profiles for $M^{2}$ when $k_{1}=0.2, S c=1.4, P r=1.5, G r=5, G m=5$ and $\tau=0.5$

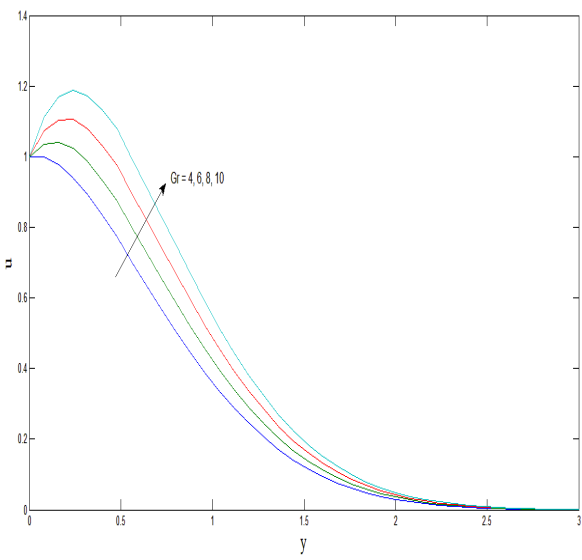

Fig. 4. Velocity profiles for $G r$ when $M^{2}=5, k_{1}=0.2, S c=1.4, P r=1.5, G m=5$ and $\tau=0.5$

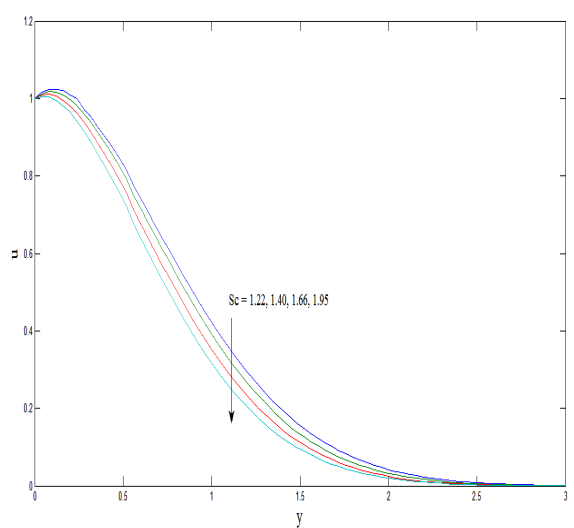

Fig. 6. Velocity profiles for $S c$ when $M^{2}=5, k_{1}=0.2, P r=1.5, G r=5, G m=5$ and $\tau=0.5$

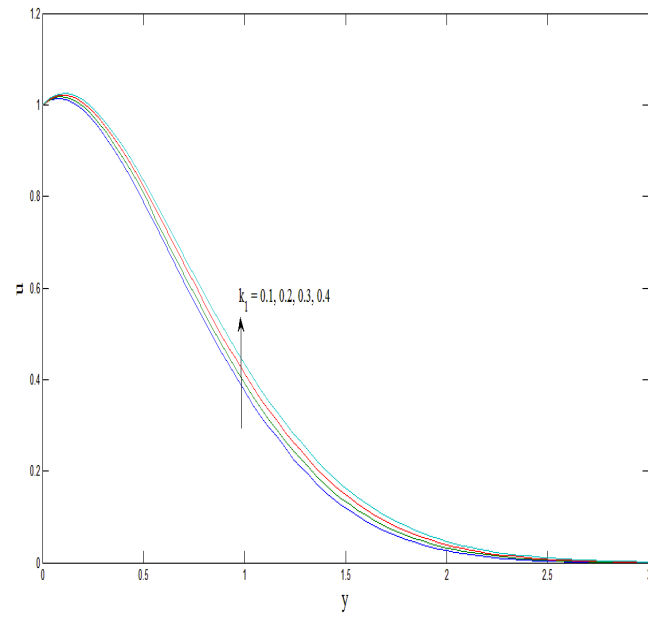

Fig. 3. Velocity profiles for $k_{1}$ when $M^{2}=5, S c=1.4, \operatorname{Pr}=1.5, G r=5, G m=5$
and $\tau=0.5$

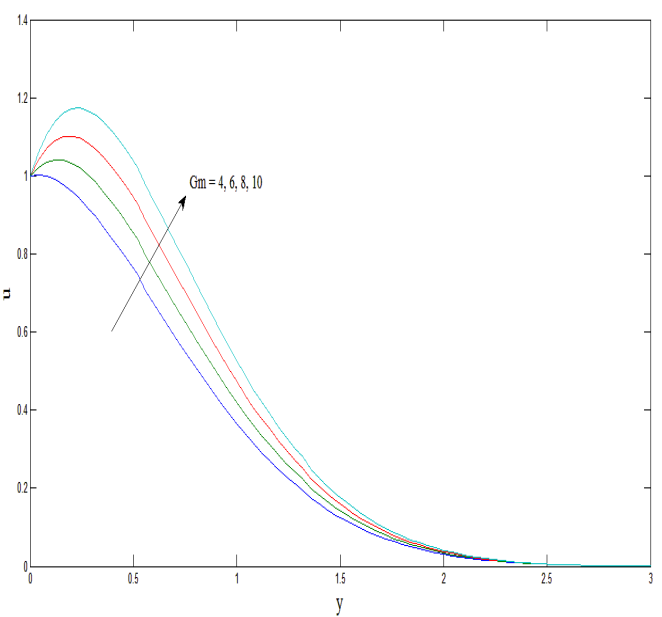

Fig. 5. Velocity profiles for $G m$ when $M^{2}=5, k_{1}=0.2, S c=1.4, P r=1.5, G r=5$ and $\tau=0.5$

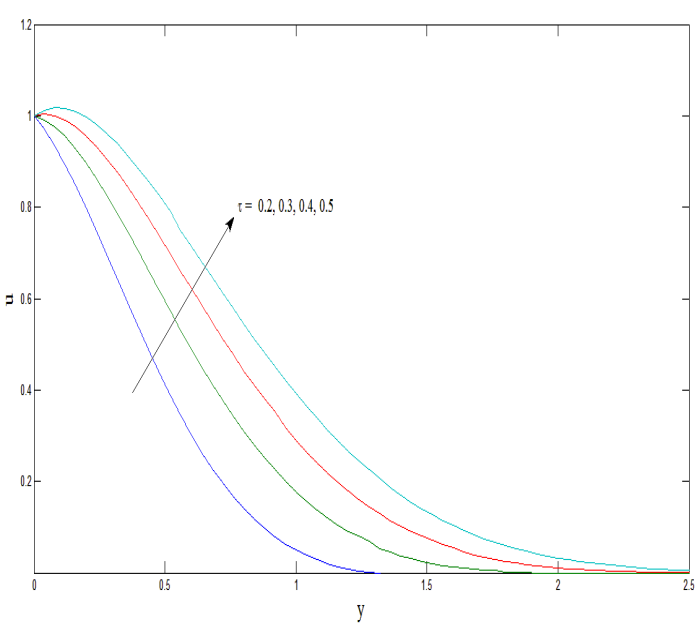

Fig.7. Velocity profiles for $\tau$ when $M^{2}=5, k_{1}=0.2, S c=1.4, P r=1.5, G r=5$ and $G m=5$ 
S. K. Ghosh et al. / JAFM, Vol. 8, No. 1, pp. 65-73, 2015.

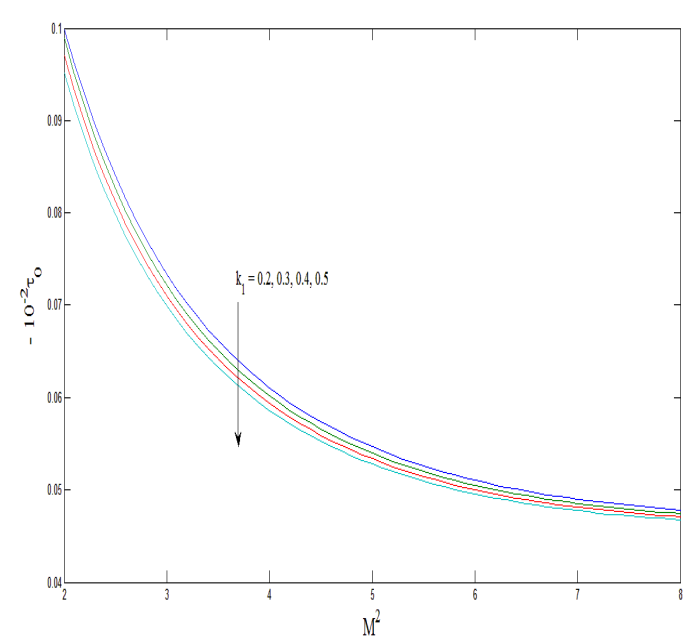

Fig. 8. Shear stress $\tau_{0}$ for $k_{1}$ when $M^{2}=5, S c=1.4, \operatorname{Pr}=1.75, G r=5$ and $\boldsymbol{G m}=\mathbf{5}$

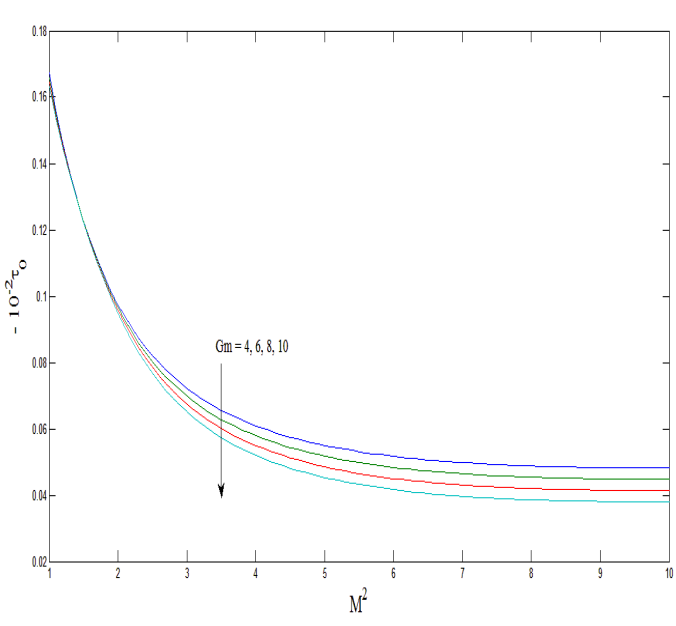

Fig. 10. Shear stress $\tau_{0}$ for $G m$ when $M^{2}=5, k_{1}=0.2, S c=1.4, P r=1.5$ and $\boldsymbol{G r}=\mathbf{5}$

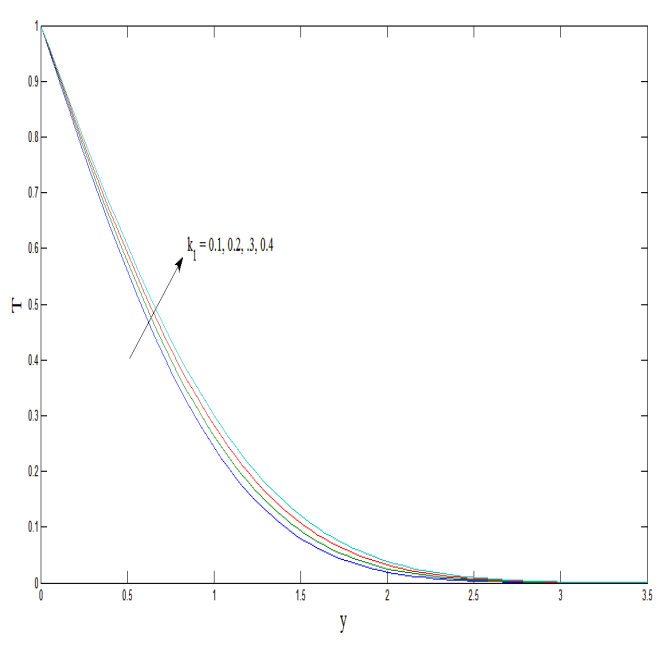

Fig. 12. Temperature profiles for $k_{1}$ when $\operatorname{Pr}=1.5$ and $\tau=0.5$

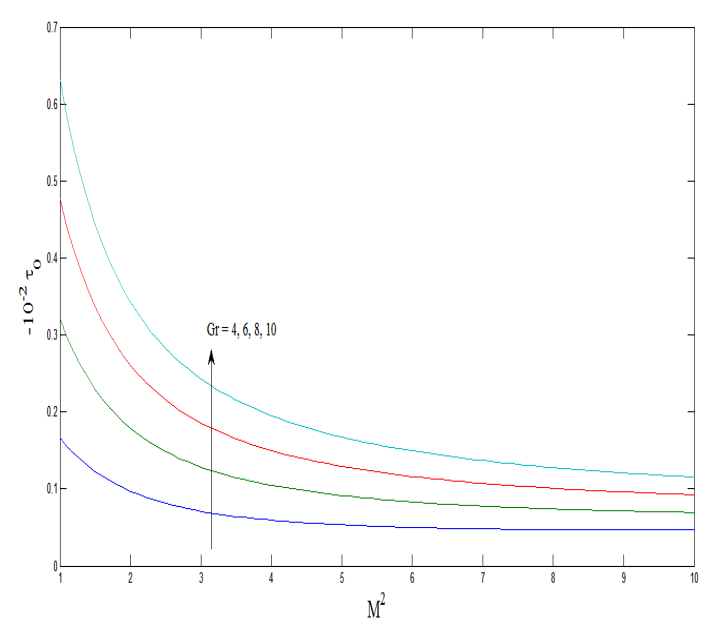

Fig. 9. Shear stress $\tau_{0}$ for $G r$ when $M^{2}=5, k_{1}=0.2, S c=1.4, P r=1.5$ and $\mathbf{G m}=\mathbf{5}$

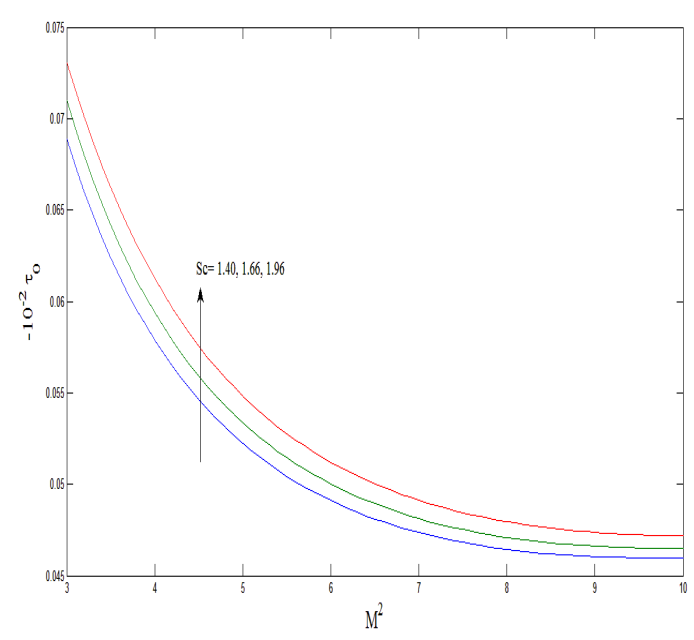

Fig. 11. Shear stress $\tau_{0}$ for $S c$ when $M^{2}=5, k_{1}=0.2, \operatorname{Pr}=1.5, G r=5$ and $\mathbf{G m}=\mathbf{5}$

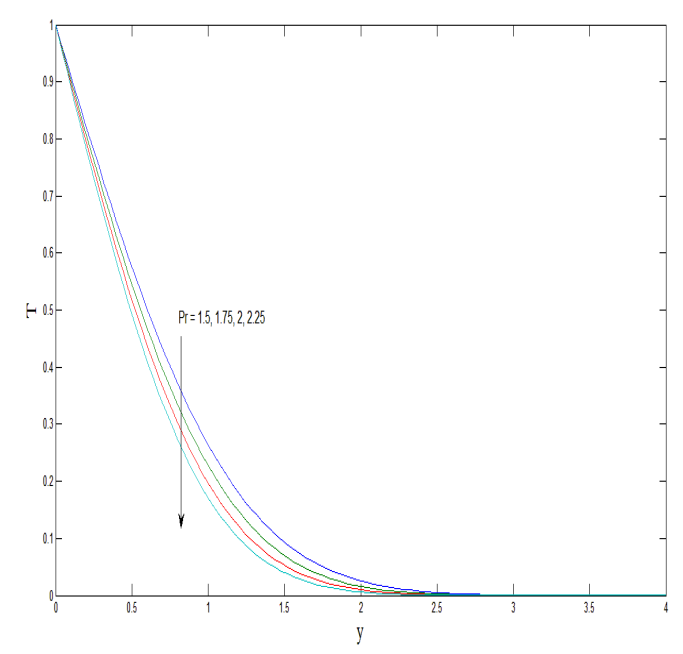

Fig. 13. Temperature profiles for $\operatorname{Pr}$ when $k_{1}=0.2$ and $\tau=0.5$ 


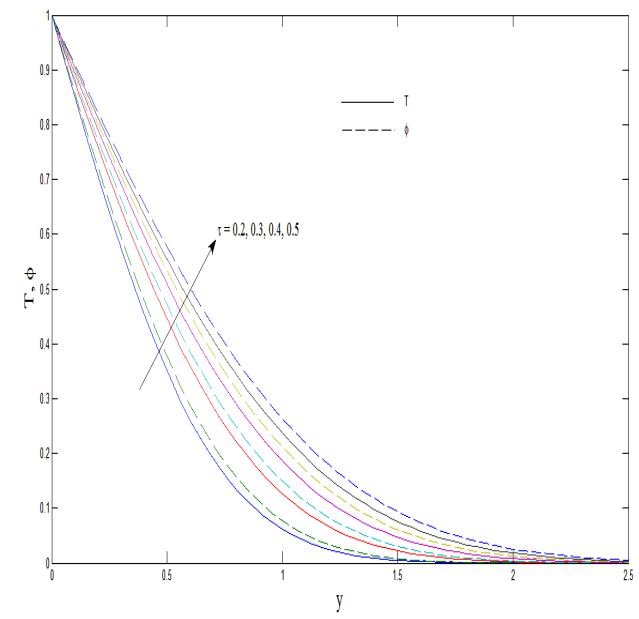

Fig. 14. Temperature and concentrattion profiles for time $\tau$ when $\operatorname{Pr}=1.5, S c=1.4$ and $k_{1}=0.2$

\section{CONCLUSION}

A mathematical analysis has been presented of the transient free convection-radiation magneto hydrodynamic viscous flow along an infinite vertical plate under a transverse magnetic field in presence of mass transfer. A flux model has been employed to simulate thermal radiation effects, valid for opticallythick gases. The dimensionless momentum, energy and

\section{REFERENCES}

Aboeldahab, E.M. and EL-Din AAG(2005). Thermal radiation effects on MHD past a semi-infinite inclined plate in the pressence of mass diffusion. Heat Mass Transfer 41(12), 1056-1065.

Ahmed, S., and K. Kalita (2013). Analytical and numerical study for MHD radiating flow over an infinite vertical surface bounded by a porous medium in presence of chemical reaction. Journal of Applied Fluid Mechanics 6(4), 597-607.

Al-Odat, M.Q. and T.A. Al-Azab (2007). Influence of chemical reaction on transient MHD free convection over a moving vertical plate. Emirates J. Engg. Research. 12(3), 15-21.

Azzam, G.(2002). Radiation effects on the MHD mixed free-forced convection flow past a semiinfinitemovingvertical plate for high temperature differences. Physics Scripts 66, 71-76.

Biberman, L.M., S.A. Medin, A.Kh. Mnatsakanian, V.N. Zatelepin,, M.B. Zhelezniak and Liubimov (1979). Radiative and Convective heat transfer in the MHD generator duct. $18^{\text {th }}$ symposium on the Engineering aspects of Magnetohydrodynamics, Butte, Monntana, USA.

Bowers, R., J. Brownell, A. Greene, D. Peterson, T. Oliphant, N. Roderick and D. Weiss (1990). Radiation magnetohydrodynamic modelling of

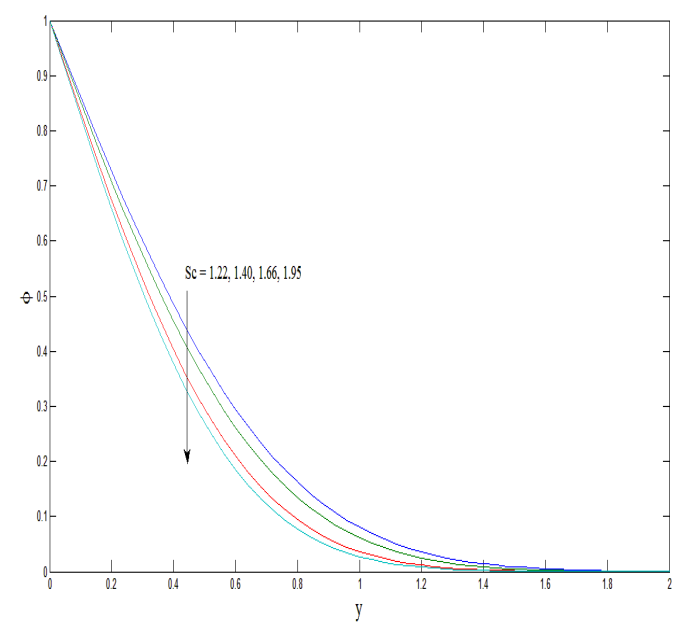

Fig. 15. Concentrattion profiles for $S c$ when $\tau=0.5$

mass conservations equations have been solved using the Laplace transform. The study has shown that the flow is accelerated with a decrease in Hartmann number. The velocity as well as the concentration decreases with an increase in the Schmidt number. Temperature of the gas is shown to be enhanced both with the elapse of time and increasing conductionradiation i.e. greater thermal radiation heat transfer contribution.

plasma flow switch performance at bhigh current levels. IEEE International conference on plasma Science, Oakland, CA, USA.

Cussler, E.L. (1998). Diffusion, Mass Transfer in Fluid Systems, 2nd edition, Cambridge University Press, Cambridge.

Duwairi, H.M., and R.A. Damseh (2004). Magnetohydrodynamic natural convection heat transfer from radiate vertical porus surface. Heat Mass transfer 40(10), 787-792.

Duwairi, H.M., and R.M. Duwairi (2005). Thermal radiation effects on MHD-Rayleigh flow with constant surface heat flux. Heat Mass Transfer 41(1), 51-57.

Ghosh, S.K., and I. Pop (2007). Thermal radiation of an optically thick gray gas in the presence of indirect natural convection. Int. J. Fluid Mechanics research 34(6), 515-520.

Helliwell, J.B., and M.F. Mosa (1979). Radiative heat transfer in horizontal magnetohydrodynamic channel flow with buoyancy effects and an axial temperature gradient. Int.J. Heat Mass Transfer 22, 657-668.

Isachenko, V.P., V.A. Osipova and A.S. Sukomel (1969). Heat Transfer, Mir Publishers, Moscow, 341-451. 
Jana, R.N., and S.K. Ghosh(2011). Radiative heat treansfer of an optically thick gray gas in the presence of indirect natural convection. World Journal of Mechanics 1, 64-69.

Mbeledogu I.U. and A. Ogulu (2007). Heat and mass transfer of an unsteady MHD natural convection flow of a rotating fluid past a vertical porous plate in the presence of radiative heat transfer. Int. J. Heat and Mass Transfer, 0, 1902-1908.

Meyer, R.C. (1958). On reducing aerodynamic heat transfer rates by magneto-hydrodynamic techniques. J. Aerospace Sciences 25, 561-566.

Patra, R.R., S. Das, R.N. Jana, and S.K.Ghosh (2012). Transient approach to radiative heat transfer free convection flow with ramped wall temperature. $J$. Applied Fluid Mechanics 5(2), 9-13.

Ram, R., and B.D. Pandey (1980). Propagation of weak MHD waves in steady hypersonic flows with radiation. AIAA Journal 18, 855-857.

Rajput, U. S., and S. Kumar (2012). Radiation effects on MHD flow past an impulsively started vertical plate with variable heat and mass transfer. Int. J. Appl. Math. Mech. 8(1), 66-85.

Rao, B. M., G. Viswanatha Reddy, M. C. Raju and S. V. K. Varma (2013). MHD transient free convection and chemically reactive flow pasta porous vertical plate with radiation and temperature gradient dependent heat source in slip flow regime. IOSR J. Applied Physics, 3(6), 22-32.

Raptis, A. and C. Perdikis (2000). MHD free convection flow by the presence of radiation. Int. Journal of Magnetohydrodynamics Plasma and Space Research 9(4), 237-246.

Raptis, A., and C.V. Masslas (1998). Magnetohydrodynamic flow past a plate by the presence of radiation. Heat and Mass Transfer 34, 107-109.

Raptis, A., C. Perdikis and H.S. Takhar (2004). Effect of thermal radiation on MHD flow. Applied Mathematics and Computation 153(3), 645-649.

Reddy, M.C. K., G. Murali, S. Sivaiah and N.V.N. Babu (2012). Heat and mass transfer effects on unsteady MHD free convection flow past a vertical permeable moving plate with radiation. I. J. Applied Mathematical Research, 12, 189-205.

Shu, Y., B.Q. Li, and K.G. Lynn (2004). Numerical modelling of internal radiation and solidification in semitransparent melts in magnetic fields. Numerical Heat Transfer, Part a, Applications 45, 957-976.

Stone, J.M., and M.L. Norman (1992). ZEUS-2D: A radiation magnetohydrodynamics code for astrophysical flows in two space dimensions. I-The hydromagnetic algorithms and tests. Astrophysical Journal (Supplement Series) 80(2), 753-790.

Takhar H. S., S. R. Gorla, V.M. Soundalgekar S.R. (1996). Radiation effects on the MHD free convection flow of a radiating gas past a semiinfinite vertical plate. Int. J. Numerical Methods heat fluid flow $6,77-83$.

Vishwakarma, J.P., R.C. Srivastava, and A. Kumar (1987). An exact similarity solution in radiation magneto-gas-dynamics for the behind a spherical shock wave. Astrphysics and Space Science 129(1), 45-52.

Yasar, O., and G.A. Moses (1992). R-MHD: an adaptive-grid radiation-magnetohydrodynamics Computer Code. Computer Physics Communications 69(2-3), 439-458. 